\title{
Prediction Models for Radiation-Induced Neurocognitive Decline in Adult Patients with Primary or Secondary Brain Tumors: A Systematic Review
}

Fariba Tohidinezhad ( $\nabla$ fariba.tohidinezhad@maastro.nl)

Maastricht University Medical Centre+: Maastricht Universitair Medisch Centrum+ https://orcid.org/0000-0001-8880-3593

Dario Di Perri

Cliniques Universitaires Saint-Luc

Catharina M.L. Zegers

Maastricht UMC+: Maastricht Universitair Medisch Centrum+

Jeanette Dijkstra

Maastricht University Medical Centre+: Maastricht Universitair Medisch Centrum+

Monique Anten

Maastricht UMC+: Maastricht Universitair Medisch Centrum+

Andre Dekker

Maastricht UMC+: Maastricht Universitair Medisch Centrum+

Wouter Van Elmpt

Maastricht UMC+: Maastricht Universitair Medisch Centrum+

Daniëlle BP Eekers

Maastricht UMC+: Maastricht Universitair Medisch Centrum+

Alberto Traverso

Maastricht UMC+: Maastricht Universitair Medisch Centrum+ https://orcid.org/0000-0001-6183-4429

Research Article

Keywords: Cranial Irradiation, Cognitive Dysfunction, Neurotoxicity, Machine Learning

Posted Date: December 14th, 2021

DOI: https://doi.org/10.21203/rs.3.rs-1151996/v1

License: @ (1) This work is licensed under a Creative Commons Attribution 4.0 International License. Read Full License

Version of Record: A version of this preprint was published at Frontiers in Psychology on March 31st, 2022. See the published version at https://doi.org/10.3389/fpsyg.2022.853472. 


\section{Abstract}

Purpose: Although an increasing body of literature suggests a relationship between brain irradiation and deterioration of neurocognitive function, it remains as the standard therapeutic and prophylactic modality in patients with brain tumors. This review was aimed to abstract and evaluate the prediction models for radiation-induced neurocognitive decline in patients with primary or secondary brain tumors.

Methods: MEDLINE was searched on October 31, 2021 for publications containing relevant truncation and MeSH terms related to "radiotherapy", "brain", "prediction model", and "neurocognitive impairments". Risk of bias was assessed using the Prediction model Risk Of Bias ASsessment Tool.

Results: Of 3,580 studies reviewed, 23 prediction models were identified. Age, tumor location, education level, baseline neurocognitive score, and radiation dose to the hippocampus were the most common predictors in the models. The Hopkins verbal learning $(n=7)$ and the trail making tests $(n=4)$ were the most frequent outcome assessment tools. All studies used regression ( $n=14$ linear, $n=8$ logistic, and $n=4$ Cox) as machine learning method. All models were judged to have a high risk of bias mainly due to issues in the analysis.

Conclusion: Existing models have limited quality and are at high risk of bias. Following recommendations are outlined in this review to improve future models: develop a standardized instrument for neurocognitive assessment in patients with brain tumors; adherence to model development and validation guidelines; careful choice of candidate predictors according to the literature and domain expert consensus; and considering radiation dose to brain substructures as they can provide important information on specific neurocognitive impairments.

\section{Introduction}

Brain tumors refers to two general types: primary malignant tumors accounting for $1 \%$ of the newly diagnosed cancer patients and secondary/metastatic brain tumors occurring in $20 \%$ of the cancer patients [1,2]. Patients with primary or metastatic brain tumors are characterized by complex and sometimes severe symptoms, usually associated with poor prognosis. Radiation Therapy (RT) is an indispensable therapeutic and prophylactic component for extending patient survival as well as effective symptom relief [3]. Depending on the location of the tumor, the use of brain RT has been confounded by the challenge of damaging critical vascular and neural structures. Patients treated with RT to the brain might experience acute irradiation triggered inflammation and be at risk for late toxicity sequelae [4]. One of the possible side effects of RT is neurocognitive decline.

Neurocognitive decline is a progressive and often disabling side effect reported in 50-90\% of the patients who receive whole brain irradiation [5]. The literature suggests that radiation-induced neurocognitive decline includes damage in multiple neural cell types, increasing neuroinflammation, reducing neurogenesis in the hippocampus, and causing functional and structural alterations in the brain blood vessels [6]. Major neurocognitive deficits including, dysfunctions related to learning, attention, memory, processing speed, spatial processing, and executive capabilities may become manifest from months to years after irradiation $[7,8]$. Improvements in radiation delivery technologies (e.g., stereotactic radiotherapy, intensity modulated radiotherapy, and proton beam therapy) allow reducing the dose delivered to the normal brain tissue $[9,10]$. Identification of the patients who might benefit from a certain treatment, will increase efficacy and potentially reduce costs.

Clinical modelling refers to the use of mathematical equations to support physicians in proposing individualized treatment indications [11]. Although medical literature overflows with articles offering to help clinicians and patients in decision making, front-line clinical use of the available prediction models remain underutilized mainly due to lack of adherence to model development and evaluation guidelines [12]. The following considerations are crucial to build a reliable prediction model: obtaining high quality multidimensional data from patients who represent the intended target population, including easy-to-use predictors which have been measured without knowledge of the outcome data, using standard outcome definition with reasonable time interval since predictor assessment, and handling statistical concerns and complexities during analysis with appropriate performance assessment [13].

Previous reviews in recent years have attempted to describe the mechanisms, impact size, and therapeutic implications of the radiation-induced neurocognitive decline in human and preclinical studies $[5,14,15]$. In this review, we aim to: 1 ) identify the prediction models for radiation-induced neurocognitive decline, 2) abstract candidate and significant predictors, and 3) discuss the quality and applicability of available models in clinical practice.

\section{Methods Search strategy}

The MEDLINE database was searched systematically to identify relevant English articles published from inception to October 31, 2021. The search strategy consisted of a combination of subject mesh terms and truncation of free words. To identify the prediction model studies, a broader version of the previously validated search strategy published by Geersing et al. [16] was combined with the terms related to "radiotherapy", "brain", and "neurocognitive impairments" (full search string provided in Supplementary Material S1). In addition, a manual search was conducted on references of the included articles. This review was carried out in accordance with the Preferred Reporting Items for Systematic Reviews and Meta-Analyses (PRISMA).

\section{Selection of eligible studies}

Studies were included if they reported the development or external validation of at least one multivariable prediction model for specific or general neurocognitive deficit in adult patients who received either therapeutic or prophylactic brain irradiation for primary or metastatic brain tumors. Two independent reviewers (FT and DDP) performed the title/abstract and full-text screening using the following exclusion criteria: 1) lack of model's specifications, 2) no significant predictors in multivariate analysis, 3) univariate associations, 4) preclinical studies, 5) editorials, letters, conference abstracts, or non-original studies, or 6) no available full text. Disagreement between reviewers was resolved by consensus. 


\section{Data extraction}

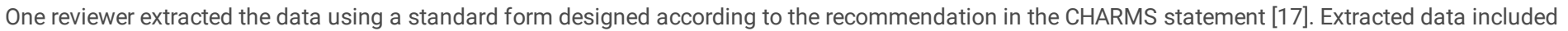

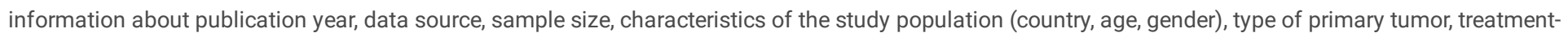

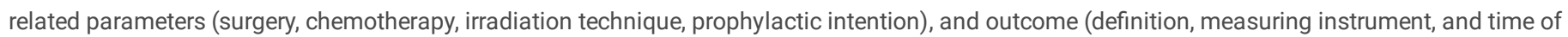

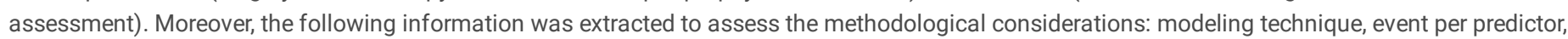

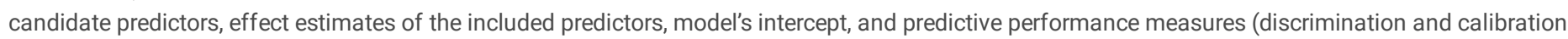
indices). A subsample of the extracted data (20\%) was checked for correctness and completeness.

\section{Quality assessment of included studies}

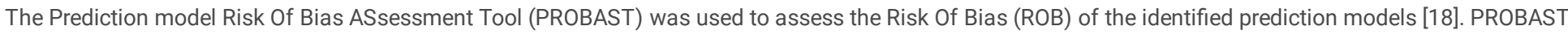

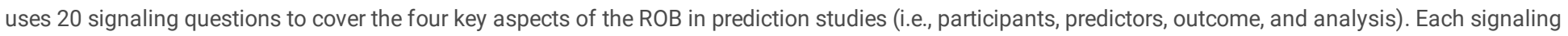

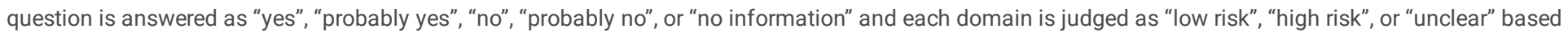

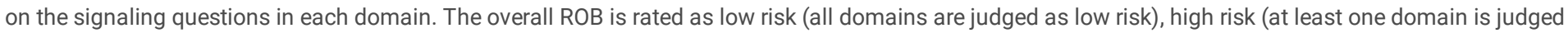

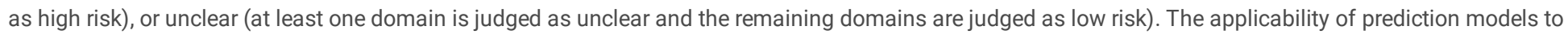
the review question was also judged as "low risk", "high risk", or "unclear" in terms of participants, predictors, and outcome.

\section{Results}

\section{General characteristics of included studies}

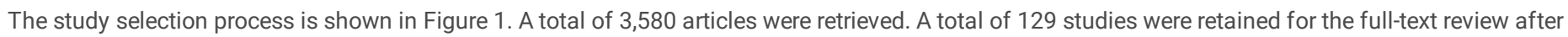

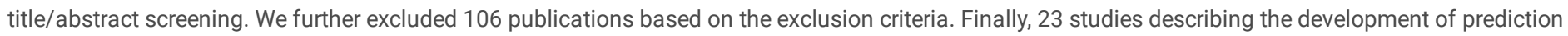

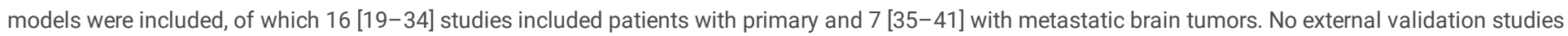
were identified.

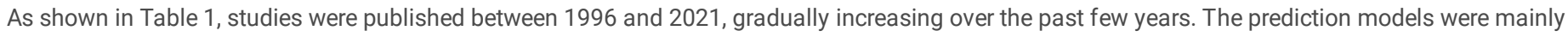

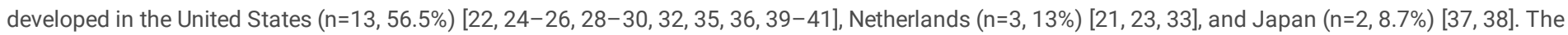

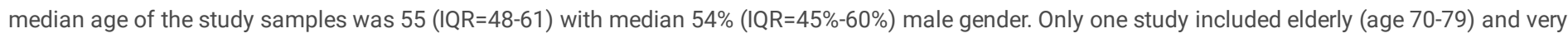
elderly patients (age $\geq 80$ ) with brain metastasis [39]. 
Table 1

Characteristics of the prediction model studies for radiation-induced neurocognitive decline in patients with primary or secondary brain tumors

\begin{tabular}{|c|c|c|c|c|c|c|c|c|c|}
\hline Study & Year & Country & $\begin{array}{l}\text { Sample } \\
\text { Size }\end{array}$ & Primary tumor type & Outcome & $\begin{array}{l}\text { Follow- } \\
\text { up }\end{array}$ & Coefficient & Prediction Equation & $\begin{array}{l}\text { Model } \\
\text { Evaluatic }\end{array}$ \\
\hline \multicolumn{10}{|c|}{ Primary brain or head \& neck tumors } \\
\hline $\begin{array}{l}\text { Gregor et al. } \\
\text { [19] }\end{array}$ & 1996 & UK & 30 & Gliomas & NART, WAIS & $\begin{array}{l}>4 \\
\text { years }\end{array}$ & OR & $\begin{array}{l}\text { (WBRT vs. Focused } \\
\text { RT } \times 7.1)^{\star}\end{array}$ & - \\
\hline $\begin{array}{l}\text { Blay et al. } \\
\text { [20] }\end{array}$ & 1998 & France & 226 & $\begin{array}{l}\text { Cerebral } \\
\text { lymphomas }\end{array}$ & Neuroimaging & $\begin{array}{l}76 \\
\text { months }\end{array}$ & $\mathrm{RR}$ & $(\mathrm{RT}+\text { chemotherapy } \times 11.5)^{\star}$ & - \\
\hline $\begin{array}{l}\text { Klein et al. } \\
\text { [21] }\end{array}$ & 2002 & Netherlands & 295 & Gliomas & SCWT & $\begin{array}{l}12 \\
\text { years }\end{array}$ & $\mathrm{RR}$ & $\begin{array}{l}\text { (Antiepileptic } \times 5.79)+ \\
(\text { tumor lateralization } \times 5.3) *\end{array}$ & - \\
\hline $\begin{array}{l}\text { Kaleita et al. } \\
\text { [22] }\end{array}$ & 2004 & USA & 79 & Brain tumors & TMT-A & NR & Beta & $\begin{array}{l}3.932+(\text { frontal } \times 1.005)+ \\
(\mathrm{GBM} \times-0.812)+(\text { Age } 36- \\
59 \times-1.174)\end{array}$ & - \\
\hline $\begin{array}{l}\text { Van Beek et } \\
\text { al. [23] }\end{array}$ & 2007 & Netherlands & 81 & Pituitary Adenoma & SF-36 & $\begin{array}{l}10 \\
\text { years }\end{array}$ & Beta & $\begin{array}{l}\text { (Radiotherapy } \times 0.56)+ \\
(\text { male } \times 0.48)+(\text { intact HPA } \\
\text { axis } \times 0.57)^{\star}\end{array}$ & - \\
\hline $\begin{array}{l}\text { Wang et al. } \\
\text { [24] }\end{array}$ & 2010 & USA & 299 & Oligodendrogliomas & MMSE & $\begin{array}{l}6.9 \\
\text { year }\end{array}$ & Beta & $\begin{array}{l}\text { (Assessment } \\
\text { timex-0.013)+(KPS 80- } \\
100 \times 2.724)+(\text { age } \\
<50 \times 1.41)^{\star}\end{array}$ & - \\
\hline $\begin{array}{l}\text { Starke et al. } \\
\text { [25] }\end{array}$ & 2011 & USA & 152 & Meningioma & Neuroimaging & 7 years & OR & $\begin{array}{l}\text { (Tumor location } \\
\text { clival/petrous } \times 4)^{\star}\end{array}$ & - \\
\hline $\begin{array}{l}\text { Gondi et al. } \\
\text { [26] }\end{array}$ & 2012 & USA & 29 & Brain tumors & WMS III WL & $\begin{array}{l}18 \\
\text { months }\end{array}$ & OR & $\begin{array}{l}\text { (D40\% of } \\
\text { hippocampus }>7.3 \\
\text { Gy } \times 19.3)^{\star}\end{array}$ & - \\
\hline $\begin{array}{l}\text { Kangas et } \\
\text { al. [27] }\end{array}$ & 2012 & Australia & 65 & Brain tumors & FACT-G & $\begin{array}{l}3.5 \\
\text { months }\end{array}$ & Beta & $\begin{array}{l}\text { Malignant×-0.23)+ } \\
\text { (baseline PCL-Sx-0.31)+ } \\
\text { (baseline FACT- } \\
\text { G/Brain×0.76)+(baseline } \\
\text { POMS depression } \times-0.46)^{*}\end{array}$ & - \\
\hline $\begin{array}{l}\text { Chapman et } \\
\text { al. [28] }\end{array}$ & 2016 & USA & 27 & Brain tumors & HVLT-PR & $\begin{array}{l}18 \\
\text { months }\end{array}$ & Beta & $\begin{array}{l}\text { (Baseline HVLT-Rx-0.62)+ } \\
\text { frontotemporalx-2.19)+ } \\
(\text { agex-0.06)* }\end{array}$ & - \\
\hline $\begin{array}{l}\text { Wong et al. } \\
\text { [29] }\end{array}$ & 2019 & USA & 198 & Brain tumors & $\begin{array}{l}\text { DS, HVLT-R, } \\
\text { COWA, TMT }\end{array}$ & $\begin{array}{l}6 \\
\text { months }\end{array}$ & OR & $(\text { Fatiguex } 1.05)^{*}$ & - \\
\hline $\begin{array}{l}\text { Gui et al. } \\
\text { [30] }\end{array}$ & 2020 & USA & 30 & GBM & HVLT-R DR & $\begin{array}{l}36.1 \\
\text { months }\end{array}$ & Beta & $\begin{array}{l}\text { (Mean dose to ipsilateral } \\
\text { hippocampus } x-0.064)+ \\
\text { (mean dose to bilateral } \\
\text { hippocampix-0.084)+ } \\
\text { (mean dose to ipsilateral } \\
\text { SVZ } x-0.089 \text { )+(mean dose } \\
\text { to bilateral SVZ } \text { S }-0.13)^{*}\end{array}$ & - \\
\hline $\begin{array}{l}\text { Dutz et al. } \\
\text { [31] }\end{array}$ & 2020 & Germany & 62 & Brain tumors & MoCA & 2 years & Beta & $\begin{array}{l}-1.16+(\text { Left } \\
\text { laterality } \times 2.37)+ \\
\text { (cerebellum anterior } \\
\text { V30Gy } \times-5.14)+ \\
\text { (cerebellum anterior } \\
\text { V40Gy } x-6.85 \text { ) }\end{array}$ & - \\
\hline $\begin{array}{l}\text { Tibbs et al. } \\
\text { [32] }\end{array}$ & 2020 & USA & 54 & Brain tumors & DKEFS-TMT & $\begin{array}{l}12 \\
\text { months }\end{array}$ & Beta & $\begin{array}{l}\text { (Beck anxiety } \\
\text { inventories } \times-0.425)^{\star}\end{array}$ & - \\
\hline $\begin{array}{l}\text { Zamanipoor } \\
\text { et al. [33] }\end{array}$ & 2021 & Netherlands & 190 & Meningioma & $\begin{array}{l}\text { DS, AVLT, } \\
\text { CWFT, CST, } \\
\text { MCT, SCWT }\end{array}$ & 9 years & OR & $\begin{array}{l}\text { (Agex } 1.024)+(\text { tumor size } \\
\text { before last intervention } \\
\times 1.022)+(\text { second } \\
\text { resection } \times 2.662)+ \\
\text { (radiotherapy } \times 2.819)+ \\
\text { (educational } \\
\text { level } \times 0.359)+(\text { years since } \\
\text { diagnosis } \times 1.130)^{\star}\end{array}$ & AUC: $0.7 \varepsilon$ \\
\hline $\begin{array}{l}\text { Langegard } \\
\text { et al. [34] }\end{array}$ & 2021 & Sweden & 266 & Brain tumors & QIQ-BN20 & $\begin{array}{l}1-3 \\
\text { months }\end{array}$ & Beta & $\begin{array}{l}\text { (Living alone×3.97) }+( \\
S C Q>4 \text { points } \times 6.71)^{\star}\end{array}$ & - \\
\hline \multicolumn{10}{|c|}{ Secondary brain tumors } \\
\hline \multirow{2}{*}{$\begin{array}{l}\text { Wolfson et } \\
\text { al. [35] }\end{array}$} & \multirow[t]{2}{*}{2011} & \multirow[t]{2}{*}{ USA } & \multirow[t]{2}{*}{75} & \multirow[t]{2}{*}{ Lung } & HVLT, COWAT, & \multirow{2}{*}{$\begin{array}{l}25.3 \\
\text { months }\end{array}$} & \multirow[t]{2}{*}{ OR } & \multirow{2}{*}{$\begin{array}{l}\text { (Treatment type } 2 \\
\text { Gy*18×8)+(treatment type } \\
1.5 \text { Gy*24×4.37)+ } \\
\text { (age } 1.12)+(\text { (education } \\
\text { level } \leq \text { High school } \\
\times 2.96)^{\star}\end{array}$} & \multirow[t]{2}{*}{-} \\
\hline & & & & & TMT-A, TMT-B & & & & \\
\hline
\end{tabular}




\begin{tabular}{|c|c|c|c|c|c|c|c|c|c|}
\hline Study & Year & Country & $\begin{array}{l}\text { Sample } \\
\text { Size }\end{array}$ & Primary tumor type & Outcome & $\begin{array}{l}\text { Follow- } \\
\text { up }\end{array}$ & Coefficient & Prediction Equation & $\begin{array}{l}\text { Model } \\
\text { Evaluatic }\end{array}$ \\
\hline $\begin{array}{l}\text { Gondi et al. } \\
\text { [36] }\end{array}$ & 2013 & USA & 583 & Lung & HVLT-R & $\begin{array}{l}12 \\
\text { months }\end{array}$ & OR & $\begin{array}{l}\text { (No prophylactic cranial } \\
\text { irradiation } \times 2.49)+ \\
\text { (baseline impairment in } \\
\text { HVLT-R } \times 3.33)+ \\
(\text { age } \leq 60 \times 2.52)^{\star}\end{array}$ & - \\
\hline $\begin{array}{l}\text { Nakazaki et } \\
\text { al. [37] }\end{array}$ & 2013 & Japan & 76 & Case-mix & MMSE & $\begin{array}{l}5.8 \\
\text { months }\end{array}$ & HR & $\begin{array}{l}\text { (Volume of the largest } \\
\text { metastasis } \times 1.102)^{\star}\end{array}$ & - \\
\hline $\begin{array}{l}\text { Yamamoto } \\
\text { et al. [38] }\end{array}$ & 2017 & Japan & 1194 & Case-mix & CTCAE v. 3 & $\begin{array}{l}46.3 \\
\text { months }\end{array}$ & $\mathrm{HR}$ & $\begin{array}{l}\text { (Age<65×1.455)+(large } \\
\text { tumor with maximum } \\
\text { diameter of largest } \\
\text { tumor } \geq 1.6 \mathrm{~cm} \times 0.375)+ \\
\text { (neurologic } \\
\text { symptoms } \times 0.413 \text { )* }\end{array}$ & - \\
\hline $\begin{array}{l}\text { Chen et al. } \\
\text { [39] }\end{array}$ & 2017 & USA & 119 & Case-mix & RTOG & $\begin{array}{l}1-3 \\
\text { months }\end{array}$ & OR & $(\text { WBRT } \times 2.82)^{*}$ & - \\
\hline $\begin{array}{l}\text { Gui et al. } \\
{[40]}\end{array}$ & 2019 & USA & 22 & Lung & HVLT-R DR & $\begin{array}{l}24 \\
\text { months }\end{array}$ & Beta & $\begin{array}{l}\text { (Absolute change in whole } \\
\text { brain volumex0.060)+ } \\
\text { (proportional change in } \\
\text { whole brain } \\
\text { volume } \times 0.79)^{\star}\end{array}$ & - \\
\hline $\begin{array}{l}\text { Brown et al. } \\
\text { [41] }\end{array}$ & 2020 & USA & 518 & Case-mix & HVLT-R & $\begin{array}{l}7.9 \\
\text { months }\end{array}$ & $\mathrm{HR}$ & $\begin{array}{l}\text { (Ages } 61 \times 0.635)+(\mathrm{HA}- \\
\text { WBRT plus } \\
\text { memantine×0.745)* }\end{array}$ & - \\
\hline
\end{tabular}

Abbreviations (alphabetic order): AUC, Area Under the Receiver Operating Characteristic Curve; AVLT, Auditory Verbal Learning Test; COWAT, Controlled Oral Word Association Test; CST, Concept Shifting Test; CTCAE, Common Terminology Criteria for Adverse Events; CWFT, Categoric Word Fluency Test; D40\%, equivalent dose in 2-Gy fractions (EQD2) assuming a/b=2 Gy to $40 \%$ of the structure volume; DKEFS-TMT, Delis-Kaplan Executive Function System-Trail Making Test; DS, Digital Span; FACT-G, Functional Assessment of Cancer Therapy-General; GBM, Glioblastoma Multiforme; HA-WBRT, Hippocampal Avoidance-Whole-Brain Radiotherapy; HPA, Hypothalamic Pituitary Adrenal; HR, Hazard Ratio; HVLT-PR, Hopkins Verbal Learning Test-Percent Retained; HVLTR, Hopkins Verbal Learning Test-Revised; HVLT-R DR, HVLT-R Delayed Recall; HVLT-R IR, HVLT-R Immediate Recall; ICD-9-CM, International Classification of Diseases 9th Clinical Modification; KPS, Karnofsky Performance Scale; MCT, Memory Comparison Test; MMSE, Mini Mental Status Examination; MoCA, Montreal Cognitive Assessment; NART, National Adult Reading Test; NR, Not Reported; OR, Odds Ratio; PCL-S, Posttraumatic stress disorder Checklist-Stressor; POMS, Profile of Mood States; QLQ-BN20, Quality of Life Questionnaire-Brain Neoplasm20; RR, Relative Risk; RT, Radiotherapy; RTOG, Radiation Therapy Oncology Group; SCQ, Self-Administered Comorbidity Questionnaire; SCWT, Stroop color-word test; SF-36, Short Form 36 Health Survey Questionnaire; SVZ, Sub-Ventricular Zones; TMT-A, Trail Making Test; UK, United Kingdom; USA, United States of America; WAIS, Weschler Adult Intelligence Scale; WBRT, WholeBrain Radiotherapy; WMS III WL, Wechsler Memory Scale-III Word List. * The intercept of the model is not reported.

Studies with primary brain tumors included the following tumor types: glioma $(n=2)[19,21]$, meningioma $(n=2)[25,33]$, cerebral lymphoma $(n=1)[20]$, glioblastoma $(n=1)$ [30], oligodendroglioma $(n=1)$ [24], and pituitary adenoma $(n=1)$ [23]. Moreover, eight studies included the patients with different types of primary brain tumors $[22,26-29,31,32,34]$. The primary tumor site of the patients with metastatic brain cancer was: lung ( $n=7)$, breast $(n=3)$, gastrointestinal $(n=3)$, kidney $(n=3)$, and skin $(n=2)$. Three studies included the patients who underwent prophylactic cranial irradiation $[35,36,40]$. Following irradiation techniques were used: intensity modulated radiotherapy $(n=4)[28,30,32,41]$, stereotactic radiotherapy $(n=4)[26,27,38,39]$, proton beam therapy $(n=3)[31$, $32,34]$, and gamma knife radiosurgery $(n=2)[25,37]$. Eleven studies included the patients who were treated with chemotherapy $[24,27,28,30-32,34-36,40$, 41].

Regarding the data source, retrospective cohort was the most popular study design used for nine studies [19, 20, 23, 27, 31, 33, 34, 37, 39], followed by prospective cohort (seven studies) [21, 22, 25, 26, 28, 30,38], and prospective trials (seven studies) $[24,29,32,35,36,40,41]$. The median sample size of the cohorts was 81 (IQR=54-266) and the median incidence of neurocognitive toxicity for studies with binary outcome was $26 \%$ (IQR=14\%-73\%). Three regression analyses were applied for model development: linear regression in 11 studies (48\%) [21-24, 27, 28, 30-32, 34, 40], logistic regression in eight studies (35\%) $[19,25,26,29,33,35,36,39]$, and Cox regression in four studies (17\%) $[20,37,38,41]$. The intercept of the model was reported in two studies [22, 31]. Among all studies, only one study performed internal validation in terms of Area Under the receiver operating characteristic Curve (AUC=0.78) [33].

\section{Variables in the prediction models}

Figure 2 presents candidate and significant predictors in the models. All variables of 23 prediction models were easily obtainable (via medical records, radiotherapy planning systems, and questionnaires), including socio-demographic, baseline comorbidities and neurocognitive functions, tumor-related variables, medication use history, and treatment-related parameters (radiotherapy, chemotherapy, and surgery). Age was the most common candidate predictor and was considered in $11(48 \%)$ prediction models followed by duration of follow-up ( $n=8,35 \%)$, tumor type $(n=5,22 \%)$, tumor location ( $n=5,22 \%)$, size/volume of brain tumor(s) $(n=5,22 \%)$, and use of radiotherapy $(n=5,22 \%)$.

The following variables were the most frequent significant predictors which remained in the models after multivariate analysis: age ( $\mathrm{n}=7,30 \%)$, tumor location $(n=5,22 \%)$, education level $(n=3,13 \%)$, baseline neurocognitive score $(n=3,13 \%)$, and radiation dose to the hippocampus ( $n=3,13 \%)$. The median number of significant predictors in prediction models was 2 (IQR=1-3). 
While radiation dose to the whole brain was removed from multivariate analysis in two prediction models [30, 38], dose to brain substructures, including hippocampus $(n=3)[26,30,41]$, subventricular zone $(n=1)$ [30], and cerebellum $(n=1)$ [31] remained significant in the prediction models.

\section{Outcome assessment}

The following tests were the most common instruments used for measuring the neurocognitive decline: Hopkins Verbal Learning Test (HVLT) ( $\mathrm{n}=7,30 \%)$ [28$30,35,36,40,41]$, Trail Making Test (TMT) $(n=4,17 \%)[22,29,32,35]$, Controlled Oral Word Association (COWA) ( $n=2,9 \%)[29,35]$, Digital Span (DS) ( $n=2,9 \%)$ $[29,33]$, and Mini-Mental State Examination (MMSE) $(n=2,9 \%)[24,37]$. Three studies (13\%) assessed the acute neurocognitive decline within the first 3 months after radiotherapy $[27,34,39]$. The remaining studies assessed long-term neurocognitive side effects with a minimum of 6 months and maximum of 12 years duration of follow-up.

\section{Risk of bias and applicability}

The results of the risk of bias and applicability assessment are shown in Table 2 and Figure 3 . All models were judged to have a high risk of bias. The most common concerning issues were seen in analysis (domain 4), including lack of model validation and inappropriate or lack of handling missing data. Several models $(n=16,70 \%)$ also had an unclear risk of bias in outcome assessment (domain 3) [19, 21, 23, 27, 29-35, 37-41]. This was due to lack of information on outcome assessment without knowledge of predictors. 
Table 2

Quality assessment for risk of bias and applicability concern of the included prediction models

\begin{tabular}{|c|c|c|c|c|c|c|c|c|c|c|}
\hline & \multirow[t]{2}{*}{ Study } & \multicolumn{4}{|l|}{ ROB } & \multicolumn{3}{|l|}{ Applicability } & \multicolumn{2}{|c|}{ Overall } \\
\hline & & Participants & Predictors & Outcome & Analysis & Participants & Predictors & Outcome & ROB & Applicability \\
\hline \multirow[t]{16}{*}{$\begin{array}{l}\text { Primary brain } \\
\text { tumors }\end{array}$} & $\begin{array}{l}\text { Gregor et al. } \\
\text { (1996) }\end{array}$ & - & + & $?$ & - & - & + & + & - & - \\
\hline & Blay et al. (1998) & - & - & - & - & + & + & - & - & - \\
\hline & $\begin{array}{l}\text { Klein et al. } \\
\text { (2002) }\end{array}$ & + & + & $?$ & - & + & + & + & - & + \\
\hline & $\begin{array}{l}\text { Kaleita et al. } \\
(2004)\end{array}$ & + & + & + & - & + & - & + & - & - \\
\hline & $\begin{array}{l}\text { Van Beek et al. } \\
\text { (2007) }\end{array}$ & + & + & $?$ & - & + & - & + & - & - \\
\hline & $\begin{array}{l}\text { Wang et al. } \\
(2010)\end{array}$ & + & $?$ & - & - & + & - & + & - & - \\
\hline & $\begin{array}{l}\text { Starke et al. } \\
(2011)\end{array}$ & - & $?$ & - & - & + & + & - & - & - \\
\hline & $\begin{array}{l}\text { Gondi et al. } \\
\text { (2012) }\end{array}$ & + & - & - & - & - & + & + & - & - \\
\hline & $\begin{array}{l}\text { Kangas et al. } \\
(2012)\end{array}$ & + & $?$ & $?$ & - & + & - & - & - & - \\
\hline & $\begin{array}{l}\text { Chapman et al. } \\
\text { (2016) }\end{array}$ & - & - & - & - & - & + & + & - & - \\
\hline & $\begin{array}{l}\text { Wong et al. } \\
\text { (2019) }\end{array}$ & + & $?$ & $?$ & - & + & + & + & - & + \\
\hline & Gui et al. (2020) & + & + & $?$ & - & + & + & + & - & + \\
\hline & $\begin{array}{l}\text { Dutz et al. } \\
(2020)\end{array}$ & + & - & $?$ & - & - & + & + & - & - \\
\hline & $\begin{array}{l}\text { Tibbs et al. } \\
(2020)\end{array}$ & + & - & $?$ & - & + & - & + & - & - \\
\hline & $\begin{array}{l}\text { Zamanipoor et } \\
\text { al. (2021) }\end{array}$ & + & - & $?$ & - & + & + & + & - & + \\
\hline & $\begin{array}{l}\text { Langegard et al. } \\
(2021)\end{array}$ & - & + & $?$ & - & - & + & - & - & - \\
\hline \multirow[t]{7}{*}{$\begin{array}{l}\text { Secondary } \\
\text { brain tumors }\end{array}$} & $\begin{array}{l}\text { Wolfson et al. } \\
\text { (2011) }\end{array}$ & + & + & $?$ & - & + & + & + & - & + \\
\hline & $\begin{array}{l}\text { Gondi et al. } \\
\text { (2013) }\end{array}$ & + & + & - & - & + & + & + & - & + \\
\hline & $\begin{array}{l}\text { Nakazaki et al. } \\
\text { (2013) }\end{array}$ & + & $?$ & $?$ & - & + & + & + & - & + \\
\hline & $\begin{array}{l}\text { Yamamoto et al. } \\
\text { (2017) }\end{array}$ & - & $?$ & $?$ & - & + & + & + & - & + \\
\hline & $\begin{array}{l}\text { Chen et al. } \\
\text { (2017) }\end{array}$ & + & + & $?$ & - & - & + & - & - & - \\
\hline & Gui et al. (2019) & + & - & $?$ & - & + & - & + & - & - \\
\hline & $\begin{array}{l}\text { Brown et al. } \\
(2020)\end{array}$ & + & - & $?$ & - & + & - & + & - & - \\
\hline
\end{tabular}

Abbreviation: ROB, Risk of bias.

+ indicates low ROB/low concern regarding applicability;

- indicates high ROB/high concern regarding applicability;

? indicates unclear ROB/unclear concern regarding applicability.

Eight models received a low score for concerns about applicability, which indicates that they are well aligned with the review question [21, 29, 30, 33, 35-38]. The remaining models had high score for concerns about applicability. This was mostly due to concerns about applicability of the participants (domain 1), which reflects the use of ungeneralizable patient populations (e.g., secondary analysis of clinical trials).

\section{Discussion}


This review summarized and evaluated 23 identified prediction models for neurocognitive decline after radiotherapy in patients with primary or metastatic brain tumors. The following risk factors were entered into at least two prediction models: age, tumor location, radiation dose to hippocampus, education level, baseline neurocognitive score, tumor type, size/volume of brain tumor(s), baseline depression/anxiety score, and type of radiotherapy (whole brain vs. focal). Although, many scholars have put substantial effort in developing prediction models for radiation-induced neurocognitive decline, the overall results are unsatisfactory. According to PROBAST, none of the models were judged to be at low risk of bias mainly due to limitations in modeling methodology.

The plethora of instruments measuring neurocognitive function is heartening. However, in the field of machine learning this negatively affects the comparability and reusability of the prediction models. There are a variety of aspects regarding the domains of instrument, how they are measured, and when specific neurocognitive functions are elicited [42]. Moreover, most of the used instruments have been developed to screen cognitive dysfunctions (e.g., dementia) in healthy elderly subjects without further adaptation and psychometric evaluation in patients with brain tumors [43]. This review clearly shows the gap in measuring the neurocognitive outcomes. Developing a standardized multidimensional instrument accompanied by an administration protocol would be beneficial toward developing a reliable prediction model.

A small number of significant predictors in prediction models (median=2) as well as the exclusion of four studies due to lack of significant predictors in multivariate analysis may imply that researchers need to follow a more systematic method for predictor selection before modeling. Although there is no recommended approach for selecting candidate predictors, using existing data in the literature in addition to a-priori knowledge of experts solicited from focus group discussions can be a solution to consider more predictive risk factors. The identified predictors in this review can be used as a potential set of predictors in future models.

Recent studies have documented deleterious associations between radiation dose to brain substructures and neurocognitive score in both pediatric and adult patients [44-48]. This is in line with the significant predictive power of radiation dose to the hippocampus, subventricular zone, and cerebellum in the available prediction models. This may provide important information about the radiation tolerance of the sub-volumes. In particular, it has been shown that equivalent doses of $2 \mathrm{~Gy}$ fractions to $40 \%$ of the hippocampus greater than $7.3 \mathrm{~Gy}$ is implicated in memory and learning impairments [26, 49]. Although current evidence on region-specific neurocognitive decline is limited, it is potentially an interesting trend for future model development studies.

In terms of the geographical distribution of the prediction models, all models were developed in countries with high human development index where early detection rate is likely higher than in developing countries [50]. Prediction models tailored to the population in less developed countries are needed before generalization and applications in clinical use.

About $26 \%, 35 \%$, and $26 \%$ of the models had a high ROB in the participant, predictor, and outcome domains, respectively. However, high ROB in the analysis domain was observed in all prediction models. Two severe deficiencies in statistical analysis were rated as high risk in the majority of studies. The first deficiency was a lack of performance assessment. Prior to applying any of these prediction models into clinical practice, clinicians need to carefully consider the predictive performance of the models in different populations. Use in clinical practice can only be considered if the performance in the local clinical population is satisfactory. The second deficiency was lack of information on handling missing data. The majority of studies did not describe the method they used to manage missing data (removing subjects, single, or multiple imputation).

About $70 \%(n=16)$ of the models used easily-obtainable predictors, which would increase their applicability to clinical practice. It is reasonable that a combination of biomarkers and baseline neurocognitive scores would improve the predictive performance of the prediction models. However, prediction models including these variables were identified as high risk in applicability since these predictors are not routinely measured in daily practice. Another high concern regarding the applicability was due to an inappropriate data source. Developing a prediction model using data which have been collected during a clinical trial may not be generalizable to the intended target population.

The following limitations should be declared: First, only English studies were included. Second, studies in non-peer reviewed literature (e.g., conference proceedings or research reports) were not considered. Third, quantitative synthesis of the effect estimates was not conducted due to the heterogeneity of outcomes.

In conclusion, 23 prediction models are available to estimate the risk of neurocognitive decline after radiotherapy in patients with primary or secondary brain tumors. The models present substantial heterogeneity in terms of outcome assessment. Moreover, the existing models were judged to have a relatively high risk of bias, with the leading limitation of lacking internal/external validation and also deficiencies in the statistical methodology for model development. For future studies it is important to carefully choose a set of candidate predictors including radiation dose to uniformly delineated brain substructures.

\section{Declarations}

Funding: The authors declare that no funds, grants, or other support were received during the preparation of this manuscript.

Conflict of Interest: The authors declare that they have no relevant financial or non-financial conflict of interest.

Ethical approval: This article does not contain any studies with human participants or animals performed by any of the authors.

Informed consent: This is a systematic review study. No informed consent is needed.

Data availability: The datasets generated during and/or analyzed during the current study are available from the corresponding author on reasonable request.

Author contributions: All authors contributed to the study conception and design. Material preparation, data extraction, and analysis were performed by Fariba Tohidinezhad and Dario Di Perri. Interpretation of the results was performed by Catharina M.L. Zegers, Jeanette Dijkstra, Monique Anten, Andre Dekker, Wouter 
Van Elmpt, and Daniëlle Eekers. Supervision was performed by Alberto Traverso. The first draft of the manuscript was written by Fariba Tohidinezhad and all authors commented on previous versions of the manuscript. All authors read and approved the final manuscript.

\section{References}

1. Siegel RL, Miller KD, Jemal A (2018) Cancer statistics, 2018. CA Cancer J Clin 68:7-30. https://doi.org/10.3322/caac.21442

2. Sacks P, Rahman M (2020) Epidemiology of Brain Metastases. Neurosurg Clin N Am 31:481-488. https://doi.org/10.1016/j.nec.2020.06.001

3. Grunert M, Kassubek R, Danz B, et al (2018) Radiation and Brain Tumors: An Overview. Crit Rev Oncog 23:119-138. https://doi.org/10.1615/CritRevOncog.2018025927

4. Tanguturi SK, Alexander BM (2018) Neurologic Complications of Radiation Therapy. Neurol Clin 36:599-625. https://doi.org/10.1016/j.ncl.2018.04.012

5. Pazzaglia S, Briganti G, Mancuso M, Saran A (2020) Neurocognitive Decline Following Radiotherapy: Mechanisms and Therapeutic Implications. Cancers (Basel) 12:146. https://doi.org/10.3390/cancers12010146

6. Makale MT, McDonald CR, Hattangadi-Gluth JA, Kesari S (2017) Mechanisms of radiotherapy-associated cognitive disability in patients with brain tumours. Nat Rev Neurol 13:52-64. https://doi.org/10.1038/nrneurol.2016.185

7. McDuff SGR, Taich ZJ, Lawson JD, et al (2013) Neurocognitive assessment following whole brain radiation therapy and radiosurgery for patients with cerebral metastases. J Neurol Neurosurg Psychiatry 84:1384-1391. https://doi.org/10.1136/jnnp-2013-305166

8. Michaelidesová A, Konírová J, Bartůněk P, Zíková M (2019) Effects of Radiation Therapy on Neural Stem Cells. Genes (Basel) 10 :E640. https://doi.org/10.3390/genes10090640

9. Scaringi C, Agolli L, Minniti G (2018) Technical Advances in Radiation Therapy for Brain Tumors. Anticancer Res 38:6041-6045. https://doi.org/10.21873/anticanres.12954

10. Weber DC, Lim PS, Tran S, et al (2020) Proton therapy for brain tumours in the area of evidence-based medicine. Br J Radiol 93:20190237. https://doi.org/10.1259/bjr.20190237

11. Chen L (2020) Overview of clinical prediction models. Ann Transl Med 8:71. https://doi.org/10.21037/atm.2019.11.121

12. Chowdhury MZI, Turin TC (2020) Precision health through prediction modelling: factors to consider before implementing a prediction model in clinical practice. J Prim Health Care 12:3-9. https://doi.org/10.1071/HC19087

13. Gu H-Q, Liu C (2020) Clinical prediction models: evaluation matters. Ann Transl Med 8:72. https://doi.org/10.21037/atm.2019.11.143

14. van Grinsven EE, Nagtegaal SHJ, Verhoeff JJC, van Zandvoort MJE (2021) The Impact of Stereotactic or Whole Brain Radiotherapy on Neurocognitive Functioning in Adult Patients with Brain Metastases: A Systematic Review and Meta-Analysis. Oncol Res Treat 1-15. https://doi.org/10.1159/000518848

15. Scoccianti S, Detti B, Cipressi S, et al (2012) Changes in neurocognitive functioning and quality of life in adult patients with brain tumors treated with radiotherapy. J Neurooncol 108:291-308. https://doi.org/10.1007/s11060-012-0821-8

16. Geersing G-J, Bouwmeester W, Zuithoff P, et al (2012) Search Filters for Finding Prognostic and Diagnostic Prediction Studies in Medline to Enhance Systematic Reviews. PLoS One 7:e32844. https://doi.org/10.1371/journal.pone.0032844

17. Moons KGM, de Groot JAH, Bouwmeester W, et al (2014) Critical Appraisal and Data Extraction for Systematic Reviews of Prediction Modelling Studies: The CHARMS Checklist. PLoS Med 11:e1001744. https://doi.org/10.1371/journal.pmed.1001744

18. Moons KGM, Wolff RF, Riley RD, et al (2019) PROBAST: A Tool to Assess Risk of Bias and Applicability of Prediction Model Studies: Explanation and Elaboration. Ann Intern Med 170:W1-W33. https://doi.org/10.7326/M18-1377

19. Gregor A, Cull A, Traynor E, et al (1996) Neuropsychometric evaluation of long-term survivors of adult brain tumours: relationship with tumour and treatment parameters. Radiother Oncol 41:55-59. https://doi.org/10.1016/s0167-8140(96)91782-x

20. Blay JY, Conroy T, Chevreau C, et al (1998) High-dose methotrexate for the treatment of primary cerebral lymphomas: analysis of survival and late neurologic toxicity in a retrospective series. J Clin Oncol 16:864-871. https://doi.org/10.1200/JC0.1998.16.3.864

21. Klein M, Heimans JJ, Aaronson NK, et al (2002) Effect of radiotherapy and other treatment-related factors on mid-term to long-term cognitive sequelae in low-grade gliomas: a comparative study. Lancet 360:1361-1368. https://doi.org/10.1016/s0140-6736(02)11398-5

22. Kaleita TA, Wellisch DK, Cloughesy TF, et al (2004) Prediction of neurocognitive outcome in adult brain tumor patients. J Neurooncol 67:245-253. https://doi.org/10.1023/b:neon.0000021900.29176.58

23. van Beek AP, van den Bergh ACM, van den Berg LM, et al (2007) Radiotherapy is not associated with reduced quality of life and cognitive function in patients treated for nonfunctioning pituitary adenoma. Int J Radiat Oncol Biol Phys 68:986-991. https://doi.org/10.1016/j.ijrobp.2007.01.017

24. Wang M, Cairncross G, Shaw E, et al (2010) Cognition and quality of life after chemotherapy plus radiotherapy (RT) vs. RT for pure and mixed anaplastic oligodendrogliomas: radiation therapy oncology group trial 9402. Int J Radiat Oncol Biol Phys 77:662-669. https://doi.org/10.1016/j.ijrobp.2009.06.004

25. Starke RM, Nguyen JH, Rainey J, et al (2011) Gamma Knife surgery of meningiomas located in the posterior fossa: factors predictive of outcome and remission. J Neurosurg 114:1399-1409. https://doi.org/10.3171/2010.11.JNS101193

26. Gondi V, Hermann BP, Mehta MP, Tomé WA (2012) Hippocampal dosimetry predicts neurocognitive function impairment after fractionated stereotactic radiotherapy for benign or low-grade adult brain tumors. Int J Radiat Oncol Biol Phys 83:e487-493. https://doi.org/10.1016/j.jjobp.2011.10.021

27. Kangas M, Tate RL, Williams JR, Smee RI (2012) The effects of radiotherapy on psychosocial and cognitive functioning in adults with a primary brain tumor: a prospective evaluation. Neuro Oncol 14:1485-1502. https://doi.org/10.1093/neuonc/nos244

28. Chapman $\mathrm{CH}$, Zhu T, Nazem-Zadeh M, et al (2016) Diffusion tensor imaging predicts cognitive function change following partial brain radiotherapy for low-grade and benign tumors. Radiother Oncol 120:234-240. https://doi.org/10.1016/j.radonc.2016.06.021

Page $9 / 13$ 
29. Wong SS, Case LD, Avis NE, et al (2019) Cognitive functioning following brain irradiation as part of cancer treatment: Characterizing better cognitive performance. Psychooncology 28:2166-2173. https://doi.org/10.1002/pon.5202

30. Gui C, Vannorsdall TD, Kleinberg LR, et al (2020) A Prospective Cohort Study of Neural Progenitor Cell-Sparing Radiation Therapy Plus Temozolomide for Newly Diagnosed Patients With Glioblastoma. Neurosurgery 87:E31-E40. https://doi.org/10.1093/neuros/nyaa107

31. Dutz A, Agolli L, Bütof R, et al (2020) Neurocognitive function and quality of life after proton beam therapy for brain tumour patients. Radiother Oncol 143:108-116. https://doi.org/10.1016/j.radonc.2019.12.024

32. Tibbs MD, Huynh-Le M-P, Reyes A, et al (2020) Longitudinal Analysis of Depression and Anxiety Symptoms as Independent Predictors of Neurocognitive Function in Primary Brain Tumor Patients. Int J Radiat Oncol Biol Phys 108:1229-1239. https://doi.org/10.1016/j.ijrobp.2020.07.002

33. Zamanipoor Najafabadi AH, van der Meer PB, Boele FW, et al (2021) Determinants and predictors for the long-term disease burden of intracranial meningioma patients. J Neurooncol 151:201-210. https://doi.org/10.1007/s11060-020-03650-1

34. Langegård U, Fransson P, Bjork-Eriksson T, et al (2021) Health-related quality of life in patients with primary brain tumors during and three months after treatment with proton beam therapy. Tech Innov Patient Support Radiat Oncol 17:5-17. https://doi.org/10.1016/j.tipsro.2021.01.004

35. Wolfson AH, Bae K, Komaki R, et al (2011) Primary analysis of a phase II randomized trial Radiation Therapy Oncology Group (RTOG) 0212: impact of different total doses and schedules of prophylactic cranial irradiation on chronic neurotoxicity and quality of life for patients with limited-disease smallcell lung cancer. Int J Radiat Oncol Biol Phys 81:77-84. https://doi.org/10.1016/j.jijrobp.2010.05.013

36. Gondi V, Paulus R, Bruner DW, et al (2013) Decline in tested and self-reported cognitive functioning after prophylactic cranial irradiation for lung cancer: pooled secondary analysis of Radiation Therapy Oncology Group randomized trials 0212 and 0214. Int J Radiat Oncol Biol Phys 86:656-664. https://doi.org/10.1016/j.jirobp.2013.02.033

37. Nakazaki K, Kano H (2013) Evaluation of mini-mental status examination score after gamma knife radiosurgery as the first radiation treatment for brain metastases. J Neurooncol 112:421-425. https://doi.org/10.1007/s11060-013-1071-0

38. Yamamoto M, Serizawa T, Higuchi Y, et al (2017) A Multi-institutional Prospective Observational Study of Stereotactic Radiosurgery for Patients With Multiple Brain Metastases (JLGK0901 Study Update): Irradiation-related Complications and Long-term Maintenance of Mini-Mental State Examination Scores. Int J Radiat Oncol Biol Phys 99:31-40. https://doi.org/10.1016/j.jijrobp.2017.04.037

39. Chen L, Shen C, Redmond KJ, et al (2017) Use of Stereotactic Radiosurgery in Elderly and Very Elderly Patients With Brain Metastases to Limit Toxicity Associated With Whole Brain Radiation Therapy. Int J Radiat Oncol Biol Phys 98:939-947. https://doi.org/10.1016/j.jjrobp.2017.02.031

40. Gui C, Chintalapati N, Hales RK, et al (2019) A prospective evaluation of whole brain volume loss and neurocognitive decline following hippocampalsparing prophylactic cranial irradiation for limited-stage small-cell lung cancer. J Neurooncol 144:351-358. https://doi.org/10.1007/s11060-019-03235-7

41. Brown PD, Gondi V, Pugh S, et al (2020) Hippocampal Avoidance During Whole-Brain Radiotherapy Plus Memantine for Patients With Brain Metastases: Phase III Trial NRG Oncology CC001. J Clin Oncol 38:1019-1029. https://doi.org/10.1200/JC0.19.02767

42. Cullen B, O’Neill B, Evans JJ, et al (2007) A review of screening tests for cognitive impairment. J Neurol Neurosurg Psychiatry 78:790-799. https://doi.org/10.1136/jnnp.2006.095414

43. Rasmusson DX, Bylsma FW, Brandt J (1995) Stability of performance on the Hopkins Verbal Learning Test. Arch Clin Neuropsychol 10:21-26

44. Haldbo-Classen L, Amidi A, Lukacova S, et al (2020) Cognitive impairment following radiation to hippocampus and other brain structures in adults with primary brain tumours. Radiother Oncol 148:1-7. https://doi.org/10.1016/j.radonc.2020.03.023

45. Toussaint L, Indelicato DJ, Stokkevåg CH, et al (2019) Radiation doses to brain substructures associated with cognition in radiotherapy of pediatric brain tumors. Acta Oncol 58:1457-1462. https://doi.org/10.1080/0284186X.2019.1629014

46. Acharya S, Guo Y, Patni T, et al (2021) Association Between Brain Substructure Dose and Cognitive Outcomes in Children With Medulloblastoma Treated on SJMB03: A Step Toward Substructure-Informed Planning. J Clin Oncol JC02101480. https://doi.org/10.1200/JC0.21.01480

47. Rodríguez de Dios N, Couñago F, Murcia-Mejía M, et al (2021) Randomized Phase III Trial of Prophylactic Cranial Irradiation With or Without Hippocampal Avoidance for Small-Cell Lung Cancer (PREMER): A GICOR-GOECP-SEOR Study. J Clin Oncol 39:3118-3127. https://doi.org/10.1200/JCO.21.00639

48. Eekers DBP, Di Perri D, Roelofs E, et al (2021) Update of the EPTN atlas for CT- and MR-based contouring in Neuro-Oncology. Radiother Oncol 160:259265. https://doi.org/10.1016/j.radonc.2021.05.013

49. Gondi V, Tomé WA, Mehta MP (2010) Why avoid the hippocampus? A comprehensive review. Radiother Oncol 97:370-376. https://doi.org/10.1016/j.radonc.2010.09.013

50. Khazaei Z, Goodarzi E, Borhaninejad V, et al (2020) The association between incidence and mortality of brain cancer and human development index (HDI): an ecological study. BMC Public Health 20:1696. https://doi.org/10.1186/s12889-020-09838-4

\section{Figures}




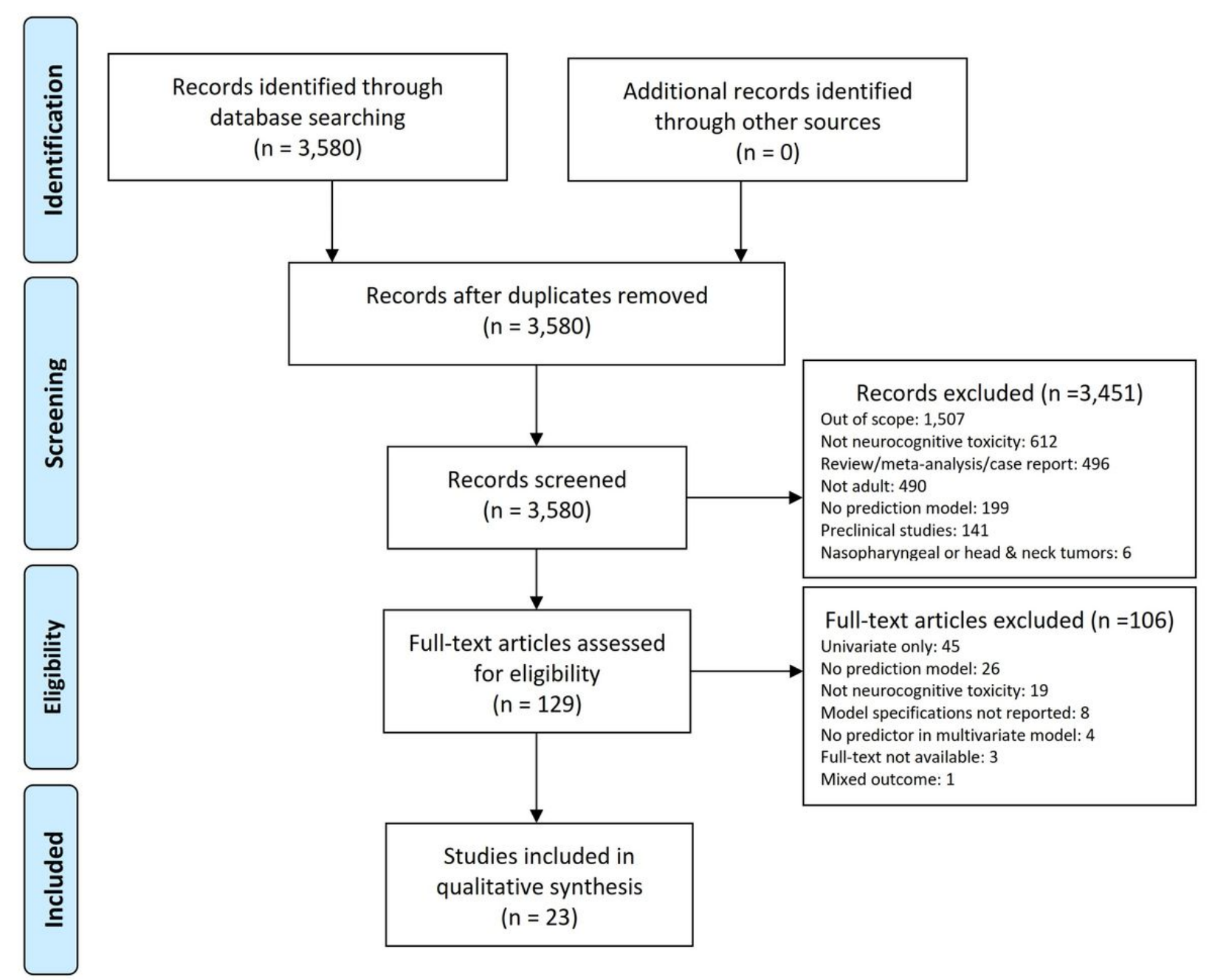

Figure 1

PRISMA flow diagram for inclusion and exclusion of studies 


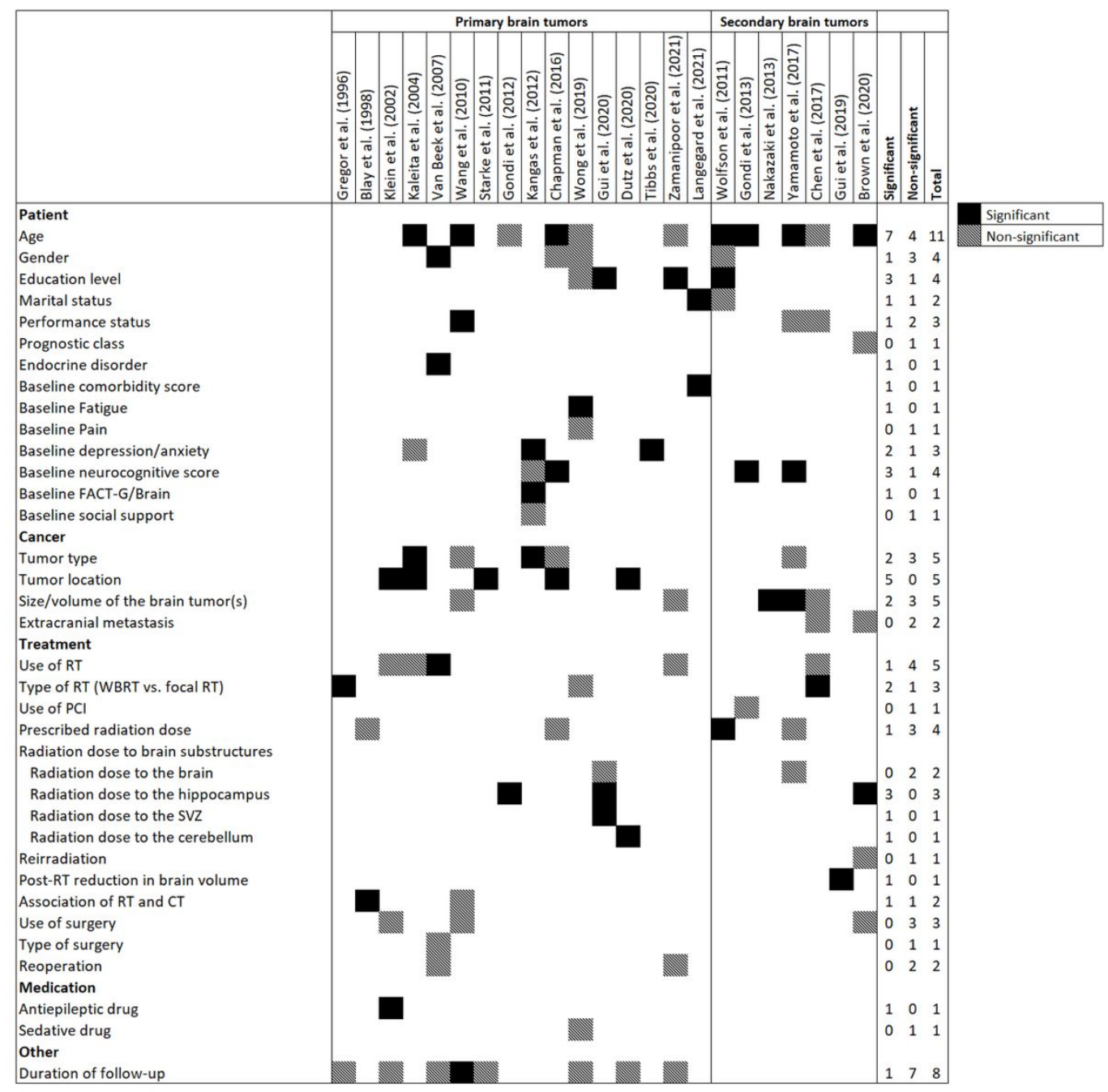

\section{Figure 2}

Frequency of candidate and significant predictors in prediction models for radiation-induced neurocognitive decline in patients with primary or secondary brain tumors Abbreviations: CT, Chemotherapy; FACT-G, Functional Assessment of Cancer Therapy-General; PCl, Prophylactic Cranial Irradiation; RT, Radiotherapy; SVZ, Subventricular Zone; WBRT, Whole brain RT. 
- Low risk $\quad$ High risk $=$ Unclear

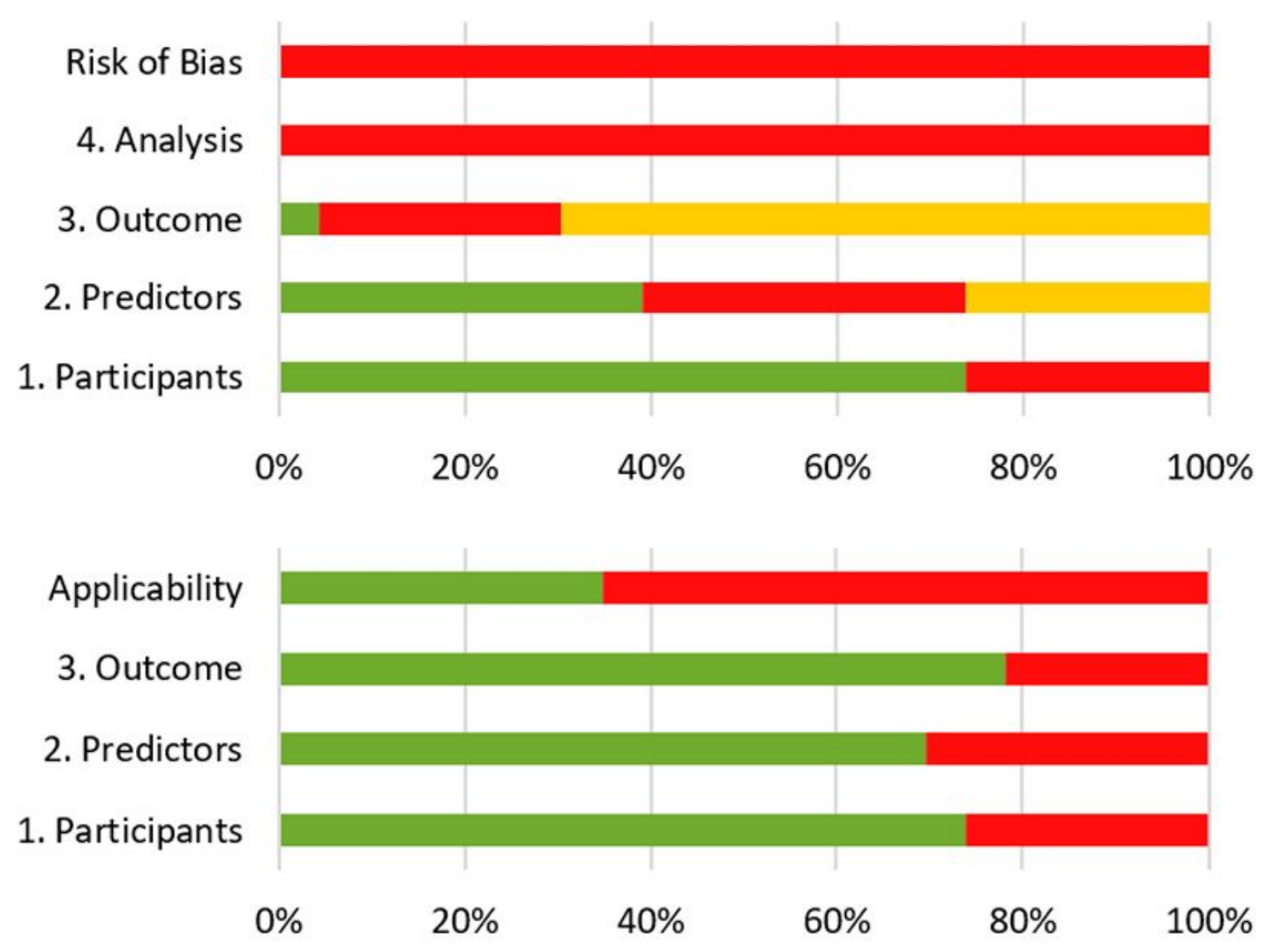

Figure 3

Summary of risk of bias (top) and applicability (bottom) according to the Prediction model Risk Of Bias Assessment

\section{Supplementary Files}

This is a list of supplementary files associated with this preprint. Click to download.

- SupplementaryMaterialS1.pdf 